\title{
Pronóstico y precipitación de los ciclones Jova (2011), Manuel (2013) y Patricia (2015) que afectaron al estado de Jalisco
}

Recibido: 14/09/2015 - Aceptado: 09/11/2015

Laura Marcela Pérez Sampablo / Claudia Leticia Martínez Robles
Maestria en Ciencia en Hidrometeorología
Universidad de Guadalajara
Luis Manuel Farfán Molina*
cICESE, Unidad La Paz
Irina Tereshchenko
Maestría en Ciencia en Hidrometeorología
Universidad de Guadalajara

\section{Resumen}

En este trabajo se presenta una evaluación de la precipitación y del pronóstico de corto plazo de tres ciclones tropicales que afectaron a Jalisco: Jova (2011), Manuel (2013) y Patricia (2015). Se evaluaron las estimaciones de precipitación de los productos de dos bases de datos derivados de sistemas de percepción remota, los cuales se compararon con las mediciones de lluvia registradas por la red de estaciones meteorológicas de superficie del Servicio Meteorológico Nacional. Adicionalmente, se analizaron las propiedades de la nubosidad mediante la combinación de imágenes infrarrojas para identificar la convección más intensa en cada ciclón. Se encontró que los sistemas de percepción remota subestiman la cantidad de lluvia registrada por las estaciones meteorológicas, y que los lugares donde los topes de las nubes son más altas y frías corresponden a la mayor producción de precipitación acumulada. Por otra parte, se analizó la calidad de los pronósticos que se emitieron en tiempo real. Para ello se determinó el error relativo y se hicieron comparaciones entre un modelo numérico y las observaciones del estado de la atmósfera. Se estableció que los errores del pronóstico se incrementaron con el paso del tiempo. De los tres ciclones examinados, Jova presentó los mayores errores de trayectoria, y la intensidad del ciclón tropical Patricia estuvo muy por debajo de los valores observados; cuando entró a tierra, la velocidad máxima sostenida fue de $259 \mathrm{~km} / \mathrm{h}$, mientras que el pronóstico fue de tan solo $185 \mathrm{~km} / \mathrm{h}$.

Palabras clave: Ciclones tropicales, pronóstico oficial, errores de pronóstico, convección, lluvia.

*Correo electrónico: farfan@cicese.edu.mx 


\title{
Forecasts and rainfall of tropical cyclones Jova (2011), Manuel (2013) and Patricia (2015) affecting the State of Jalisco
}

\author{
Laura Marcela Pérez Sampablo / Claudia Leticia Martínez Robles \\ Maestría en Ciencia en Hidrometeorología, Universidad de Guadalajara \\ Luis Manuel Farfán Molina* \\ CICESE, Unidad La Paz \\ Irina Tereshchenko \\ Maestria en Ciencia en Hidrometeorología \\ Universidad de Guadalajara
}

\begin{abstract}
In this work an evaluation of precipitation and short-term rainfall estimations of three Tropical Cyclones (TCs) that affected the State of Jalisco, Jova (2011), Manuel (2013) and Patricia (2015) is presented. Rainfall estimations from two remote sensing databases were evaluated and compared against rainfall registered by the meteorological stations network of the Meteorological National Service (Servicio Meteorologico Nacional, smn). Additionally, satellite imagery was used to analyze cloud properties by combining infrared images to identify the deepest convection in each of the TCs considered. It was found that remote sensing systems underestimate the amount of rainfall registered in the meteorological stations and that those places where the cloud-tops are higher and colder correspond to the highest accumulated rainfall amounts. In addition, the quality of the real time emitted forecasts was analyzed. For this, relative errors were estimated and a comparison between a numerical model and the observed atmospheric conditions was made. It was determined that forecast errors increased as time passed. Of the three analyzed cyclones, tropical cyclone Jova presented the largest trajectory error and the intensity forecast for tropical cyclone Patricia was below the observed values, when it made landfall, maximum sustained wind speed was $259 \mathrm{~km} / \mathrm{h}$ while the forecast was only $185 \mathrm{~km} / \mathrm{h}$.
\end{abstract}

Key words: Tropical cyclones, official forecast, forecast errors, convection, rainfall.

*E-mail: farfan@cicese.edu.mx 


\section{Introducción}

Los ciclones tropicales (ст) son sistemas de circulación que se desarrollan sobre aguas tropicales y subtropicales (Anthes, 1982). Estos fenómenos, debido a sus condiciones meteorológicas, traen consigo principalmente intensas precipitaciones, oleaje alto y fuertes vientos, los cuales, en conjunto, pueden provocar inundaciones en las zonas bajas o de planicie, desplazamiento de personas, disminución de la producción de alimentos y cambios temporales en el entorno.

Para el pronóstico de los ст se requiere la predicción de su trayectoria y de su intensidad (Holland, 2009). Para la realización de cada pronóstico se emplean diferentes técnicas: estadísticas, modelos determinísticos, modelación numéricadinámica, estadística-dinámica, teoría de conjuntos o técnicas híbridas (Roy y Kovordányi, 2012; Heming y Goerss, 2010). Los pronósticos normalmente se emiten cada seis horas $(0,6,12$ y 18 horas del meridiano de Greenwich), pero cuando un ciclón amenaza a tierra se incrementa el número de avisos con frecuencia de tres horas, con un alcance de 120 horas (cinco días). Una vez que se emiten los pronósticos de trayectoria e intensidad se evalúa el rendimiento de estos y se calcula el error.

Los pronósticos de trayectoria e intensidad han sido desarrollados y mejorados con el paso del tiempo para varias cuencas oceánicas desde 1980. Entre los grupos de investigación que emiten pronósticos para las diferentes cuencas están el Servicio Meteorológico Nacional (smN) (cuenca del Pacífico norte oriental, México) y el Centro Nacional de Huracanes (NHC, por sus siglas en inglés). Los pronósticos que más se usan son el Pronóstico Oficial (OFCL), GFs (Global Forecast System) y CLIPER (Climatology and Persistence), entre muchos otros. Primordialmente, el ofCL es en el que se basan los tomadores de decisiones para realizar las alertas y a veces las evacuaciones de las personas.

Los ст pueden generar diversos efectos sobre la zona costera y lugares aledaños por donde pasan, como la marea de tormenta en las costas bajas y el oleaje en las costas profundas, los vientos y las lluvias intensas. Mientras que para zonas que se encuentran a cientos de kilómetros de la costa el efecto dominante es la precipitación intensa; estas lluvias resultan muy relevantes debido a su impacto socioeconómico, ya que son capaces de mitigar las graves consecuencias de la temporada de sequía, especialmente en el sector agrícola; pero cuando se producen por periodos largos y en lugares que por su localización geográfica 
son propensos a inundaciones y deslaves, pueden conducir a desastres en áreas densamente pobladas (Farfán, D’Sa, Liu y Rivera-Monroy, 2014). Aquí hay que señalar que las lluvias acumuladas asociadas a los ст no dependen de la intensidad de estos últimos, pues se ha observado que muchos sistemas débiles como las depresiones tropicales también pueden producir grandes cantidades de lluvia.

Por otro lado, la dinámica poblacional de las zonas costeras de México indica un desplazamiento de las poblaciones humanas hacia ellas, como lo observado en 2005, cuando el número de habitantes de todos los estados costeros del país fue de 47344 698, 2.7 millones más que en el año 2000, y se estima que para 2030 la población costera aumente a 55 millones (Partida Bush, 2006). México es afectado por los ct tanto por la cuenca del Atlántico de junio a noviembre, como por el Pacífico de mayo a noviembre. Para el caso del Pacífico mexicano (figura 1), durante el periodo 2007-2015 se presentaron 129 eventos ciclónicos que incluyen tormentas tropicales (тт) y huracanes (н), de los cuales 17 entraron a tierra.

Recientemente, el estado de Jalisco fue golpeado por eventos ciclónicos de gran importancia: Jova (2011), Manuel (2013) y Patricia (2015). Debido a que esta entidad ostenta una orografía variada, las mayores afectaciones por el paso de los ст se producen sobre todo en la zona costera y en la zona montañosa cercana a la costa. El territorio jaliscience se ubica sobre la Sierra Madre Occidental, la Sierra Madre del Sur, el Eje Neovolcánico y la Mesa del Centro, sistemas montañosos que podrían constituir una barrera contra algunos hidrometeoros intensos. Comparado con las demás costas, Jalisco tiene $3.2 \%$ del total nacional, Baja California Sur 19.2\%, con 2131 km de litoral, y Quintana Roo 1176 km, lo que significa $10.6 \%$ del total nacional (Farfán et al., 2014).

El atractivo común entre los tres estados es la zona costera, que representa una gran fuente de ingresos económicos gracias al turismo. Sin embargo, ninguno está exento de sufrir el impacto de al menos un hidrometeoro, que puede ser desde una tormenta tropical hasta un huracán mayor.

En este trabajo se describirán y evaluarán los pronósticos de trayectoria e intensidad de los tres ст antes mencionados, además se evaluará la cantidad de lluvia durante estos eventos usando sistemas de percepción remota TRMm y PERSIANN y datos observados. 


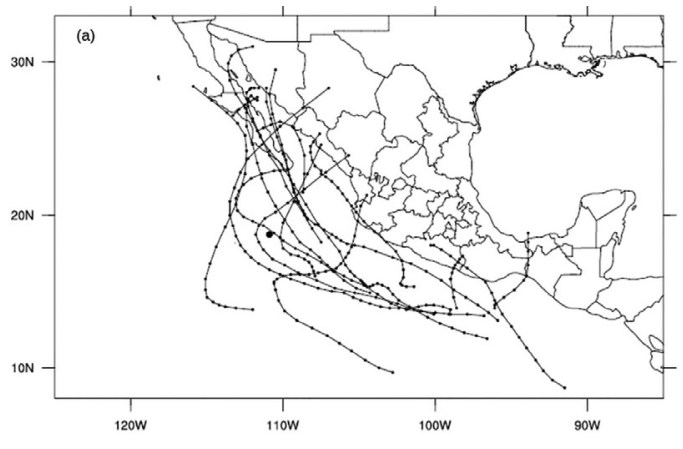

a

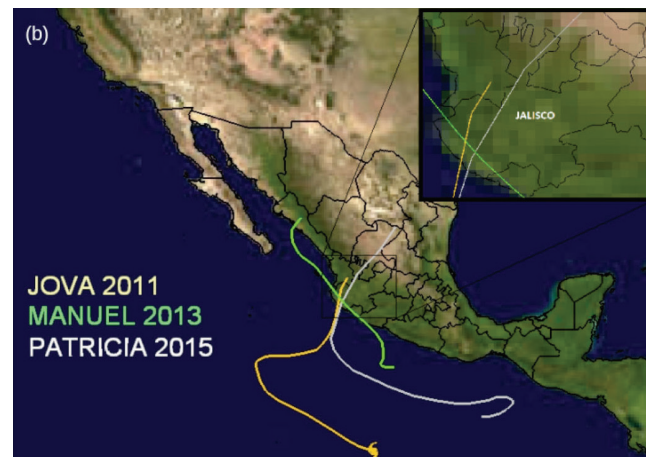

$\mathrm{b}$

Fuente: Elaboración propia en el software Integrated Data Viewer (IDv).

Figura 1. Trayectoria de los 17 ct que entraron a tierra

a) Durante la temporada 2007-2015 en el Pacífico mexicano,

b) Trayectoria de Jova (2011), Manuel (2013) y Patricia (2015)

\section{Antecedentes}

Los ст han sido abordados desde diferentes perspectivas: las causas que los generan, su formación, estructura, intensidad, así como los daños que ocasionan a su paso, tanto a los habitantes de las comunidades como al ambiente. Para el estudio de estos fenómenos se emplean diversas herramientas, por ejemplo la información de las estaciones meteorológicas automáticas que miden las variables atmosféricas de temperatura, velocidad del viento y lluvia principalmente, además se utilizan imágenes satelitales de nubosidad, precipitación, temperatura del mar y en algunas ocasiones aviones de reconocimiento.

Para el caso de estudios de evaluación de pronósticos en nuestro país se encuentran los realizados por Rosengaus (2001), quien analizó la temporada 2000 de ст en México para el Atlántico y el Pacífico. Reportó que a pesar de los mejores datos empleados en el Atlántico, el desempeño de los pronósticos en el Pacífico es ligeramente mejor, lo cual se debe al comportamiento más climatológico de los ciclones en esta cuenca. 
Se necesita contar con todas las herramientas disponibles para perfeccionar tanto los pronósticos de trayectoria como las estimaciones de los sistemas de percepción remota a fin de contribuir a optimizar la planificación y acciones de prevención ante los posibles desastres naturales que puedan ocurrir debido al impacto de los ст sobre tierra firme en territorio mexicano.

En el trabajo de Colín Olivares, Chimal Vázquez e Ihl (2015) se mostró que hay tendencia a aumentar la incidencia de huracanes y tormentas por el Atlántico, mientras que Farfán, D’Sa, Liu y Rivera-Monroy (2014) señalaron que por el lado del Pacífico también existen frecuentes impactos de cт, especialmente en Baja California Sur, teniendo el primer lugar en estos impactos, en tanto que Jalisco está clasificado como el onceavo estado más azotado por ст incluyendo huracanes mayores durante el periodo 1970-2010.

\section{Descripción general e impacto de cada ciclón}

Jova (2011)

Jova (figura 1b) se desarrolló del 6 al 12 de septiembre sobre el Pacífico nororiental y sur de México; la máxima intensidad que registró fue categoría 3 de la escala de Saffir-Simpson y tocó tierra como categoría 2. Jova fue un ciclón cuya trayectoria inició a más de $1000 \mathrm{~km}$ al sur de la costa de Colima, de ahí se desplazó hacia el noroeste y posteriormente recurvó (es decir, cambió de dirección con desplazamiento hacia el este) hacia el noroeste con rumbo a la costa occidental de México, lugar en el que tocó tierra como categoría 2 a la 1:00 horas a $8 \mathrm{~km}$ al sur de la población de La Fortuna, Jalisco, donde presentó vientos máximos sostenidos de $160 \mathrm{~km} / \mathrm{h}$ y rachas de $195 \mathrm{~km} / \mathrm{h}$.

Las peores afectaciones ocurrieron en varios municipios de Colima, y el SMN reportó que los estados de Michoacán y Nayarit también fueron afectados (Bravo Lujano y Hernández Unzón, 2011), mientras que el NHc indicó que la lluvia torrencial asociada ocasionó deslaves de cerros y desbordamientos de ríos dejando nueve muertos (Brennan, 2012). Además, 107000 personas se quedaron sin servicio eléctrico y 2700 fueron evacuadas. Hubo inundaciones en Cihuatlán, Melaque y Barra de Navidad, todas poblaciones de Jalisco. 


\section{Manuel (2013)}

Manuel (figura 1b) se desarrolló del 13 al 19 de septiembre, con categoría 1 en la escala de Saffir-Simpson y tocó tierra dos veces, la primera en la costa sur de México como тт y posteriormente en la costa norte sobre el estado de Sinaloa como categoría 1. Manuel no fue un huracán muy intenso, pero aportó una cantidad considerable de humedad al país durante su paso por varios estados. Casi la totalidad de la nación sufrió los efectos de la lluvia asociada y los máximos de precipitación se produjeron en la zona montañosa, aunque en la zona costera hubo precipitaciones importantes, como se verá más adelante. Los deslaves e inundaciones repentinas ocasionadas por la acumulación prolongada de lluvia causaron la muerte de dos personas en Jalisco, y en Guerrero ocurrieron 97 muertes debidas a un deslave que afectó a la población de La Pintada (Pasch y Zelinsky, 2014). Se estima que las pérdidas económicas originadas por Manuel fueron de aproximadamente 42000 millones de dólares, siendo Guerrero el estado más afectado con las mayores pérdidas.

\section{Patricia (2015)}

Patricia (figura 1b) se desarrolló del 22 al 24 de octubre. Es el huracán más reciente que impactó a Jalisco, con categoría 5. El NHc reportó que Patricia se intensificó muy rápido sobre aguas anormalmente cálidas (Kimberlain, Blake y Cangialosi, 2016), ya que en cuestión de unas horas saltó de categoría 2 a 4 y 5 y finalmente arribó a las costas de Jalisco el 23 de octubre a las 18:00 horas, tiempo del centro, según lo reportado por la Comisión Nacional del Agua (Conagua).

La trayectoria que siguió este huracán es muy parecida a la de Kenna (2002), que tocó tierra en San Blas, Nayarit. Sin embargo, sus efectos se dejaron sentir también en Puerto Vallarta, Jalisco. Se registró que Patricia impactó en la localidad llamada Emiliano Zapata (Nájar, 2015), al suroeste de Jalisco, casi en los límites con Colima. La figura 6, más adelante, muestra las similitudes entre las trayectorias de Kenna (2002) y Patricia (2015).

\section{Patrones de convección}

Aquí se explica brevemente cómo se utilizaron las imágenes infrarrojas del satélite geoestacionario GOES-15 para observar algunas propiedades de la nubosidad, 
distribución y temperaturas a fin de determinar dónde se produce la convección más intensa y los lugares que fueron afectados por esta.

La figura 2 exhibe el resultado de combinar cientos de imágenes en la banda infrarroja. Se puede apreciar la trayectoria del huracán desde su formación hasta el impacto; los tonos oscuros denotan la nubosidad asociada a los ст: a) Jova (2011), b) Manuel (2013) y c) Patricia (2015), y estos, de acuerdo con la escala de colores en la imagen, representan la temperatura de las nubes; las nubes bajas $(\sim 7 \mathrm{~km})$ van del color púrpura al verde, mientras que las nubes altas ( 13-16 km) del amarillo al rojo oscuro.

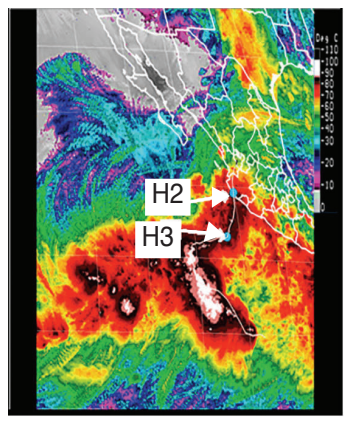

a)

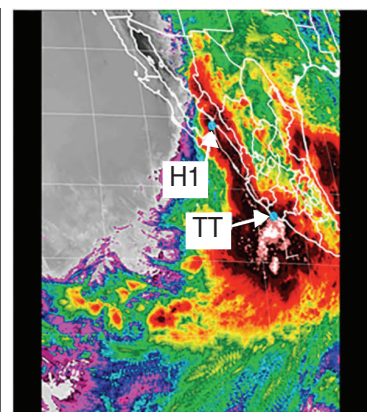

b)
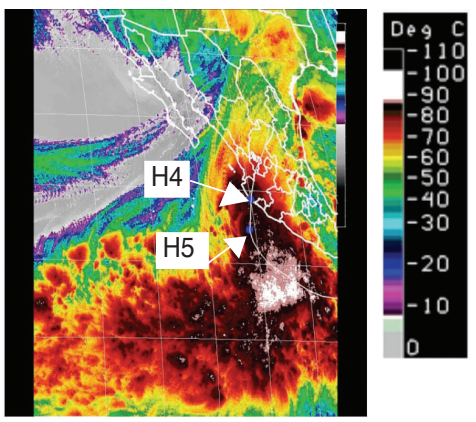

c)

Fuente: Elaboración propia usando IDv.

FiguRa 2. Patrones de la convección y nubosidad asociada a los ct considerados: a) Jova (2011), b) Manuel (2013) y c) Patricia (2015)

Existen nubes que pueden llegar a los $19 \mathrm{~km}$ de altura, y son regiones que indican dónde hay convección profunda y se puede producir la lluvia y vientos más intensos en un cт. Por eso es necesario seguir la evolución de la nubosidad cuando un sistema se acerca a tierra, pues si bien la convección profunda se da principalmente en el mar, puede haber casos en los que se presente convección importante también sobre tierra firme, lo cual, dependiendo del rango de acción del Ст, puede ser catastrófico en la zona costera o en lugares propensos a sufrir deslaves de tierra, desbordamiento de ríos, etcétera.

La convección más intensa (regiones de pixeles blancos) que se observa en la figura 2 se produce en el mar y también es el lugar donde los topes de las nubes más altas tienen temperaturas muy bajas $\left(-100^{\circ} \mathrm{C}\right.$ o menores), de acuerdo con la escala de colores, y esos pixeles fríos, según el trabajo de Ebert y Holland 
(1990), son indicadores de nubes cuyas alturas pueden llegar a $19 \mathrm{~km}$. Los puntos azules en cada figura señalan dónde fue la intensidad máxima de los huracanes considerados: a) Jova (2011) tuvo categoría 3 (H3) en el mar pero impactó a Jalisco con categoría 2 (H2) en la escala Saffir-Simpson; b) Manuel (2013) impactó como тт en Michoacán, se disipó por un breve tiempo pasando sobre Jalisco pero se intensificó nuevamente en el Golfo de California impactando Sinaloa con categoría 1 (H1); en esa misma figura se advierte otra región de convección intensa por el lado del Golfo de México: se trata del huracán Ingrid (2013), que también impactó al país. Ingrid estuvo activa del 12 al 17 de septiembre, según lo reportado por el NHc (Pasch y Zelinsky, 2014). La figura 3c) muestra al huracán Patricia (2015) que adquirió su máxima intensidad (H5) en el mar y tocó tierra firme con categoría 4 (H4) en la escala Saffir-Simpson, de acuerdo con lo reportado por la Conagua.

Comparando las estimaciones de TRMM y PERSIANN con las imágenes infrarrojas, se puede observar que ambas distribuciones espaciales de la precipitación estimada coinciden bastante bien con la distribución de la nubosidad asociada a los tres ст, aunque es de menor calidad la estimación de PERSIANN.

La figura 3 muestra un acercamiento de las imágenes infrarrojas hacia la región de Jalisco, en la cual se aprecian las estaciones meteorológicas convencionales representadas por " $\mathrm{X}$ ” y que registraron la precipitación asociada a los huracanes mientras se desplazaban tierra adentro.

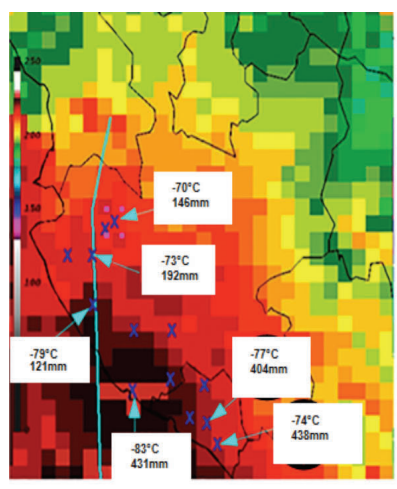

a)

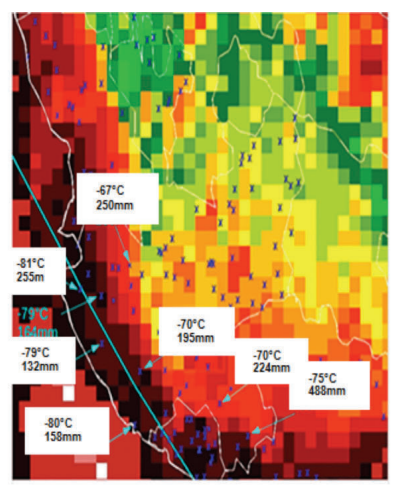

b)

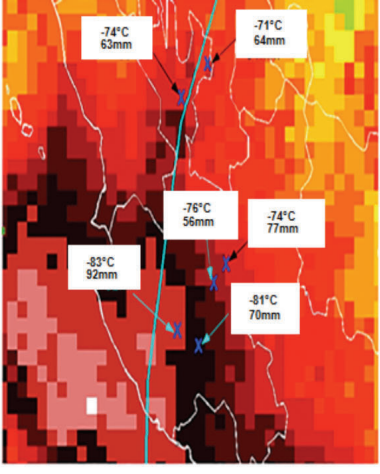

c)

Fuente: Elaboración propia usando IDv.

Figura 3. Imágenes combinadas de satélite G0ES-15 para: a) Jova (2011), b) Manuel (2013) y c) Patricia (2015) 
Las regiones oscuras constituyen los lugares donde los topes de las nubes tuvieron temperaturas de -70 a $-80^{\circ} \mathrm{C}$, según la escala de colores en la figura 3 , y las trayectorias de cada ст se representan con la línea azul clara. Los números azules indican la temperatura $\left({ }^{\circ} \mathrm{C}\right)$ de los topes de las nubes que se encontraban sobre las estaciones meteorológicas y la lluvia acumulada ( $\mathrm{mm}$ ) que estas registraron durante cada evento. Por ejemplo, para Jova (2011) se puede apreciar que hay dos máximos importantes de lluvia en el estado de Colima, de $404 \mathrm{~mm}$ donde la temperatura de las nubes fue de $-77^{\circ} \mathrm{C}$, y de $438 \mathrm{~mm}$ donde la temperatura fue de $-74^{\circ} \mathrm{C}$. De manera similar se puede ver la información para Manuel (2013) y Patricia (2015) en las figuras 3b y 3c, respectivamente, aunque en este último caso fueron pocas las estaciones que registraron la lluvia asociada. Los máximos se observaron al lado derecho del sistema y sobre todo en la zona costera.

\section{Patrones de lluvia acumulada}

Las estimaciones realizadas por TRMM y PERSIANN se procesaron mediante el software Integrated Data Viewer (IDv) y a continuación se muestran las diferencias entre cada sistema de percepción remota.

Jova (2011)

Ambas estimaciones de lluvia presentan una distribución espacial distinta, los productos exhiben los mismos intervalos de contornos (mínimo 100, máximo 500), lo único que tienen en común es la trayectoria observada, como puede advertirse en la figura 4 .

\section{Manuel (2013)}

Manuel no fue un huracán muy intenso, pero aportó una cantidad considerable de humedad al país durante su paso por varios estados. Muchas poblaciones sufrieron los efectos de la lluvia asociada a Manuel, como lo muestra la figura 5 con las estimaciones de la lluvia acumulada hechas por TRMM y PERSIANN del 13 al 19 de septiembre de 2013 . 


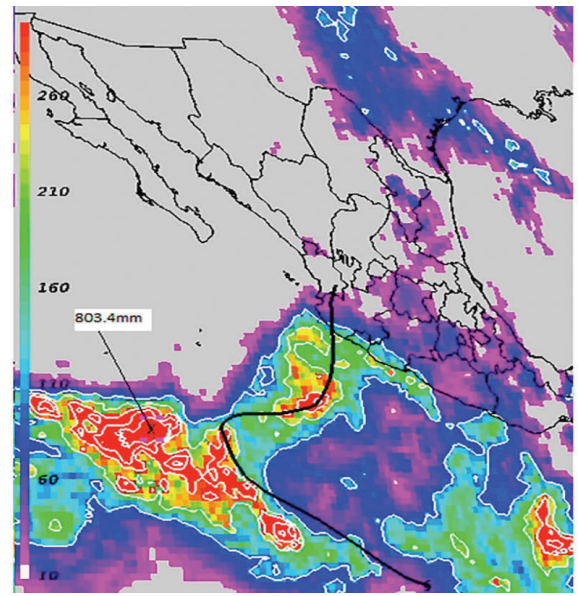

a)

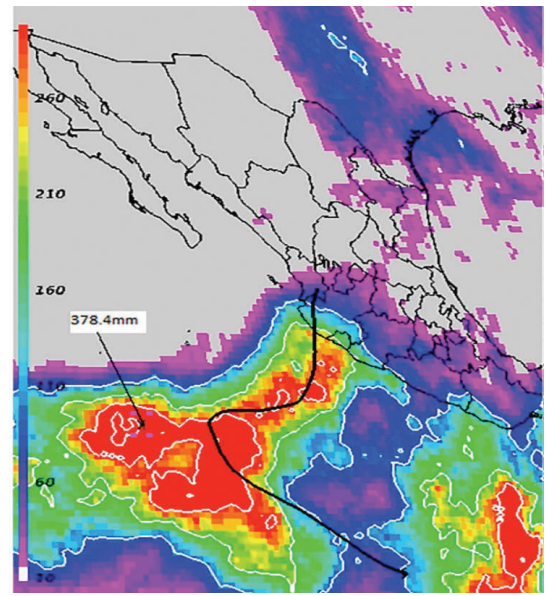

b)

Fuente: Elaboración propia usando IDv.

Figura 4. Estimación de lluvia usando a) TRMm y b) PERSIANn para el huracán Jova del 6 al 12 de octubre de 2011

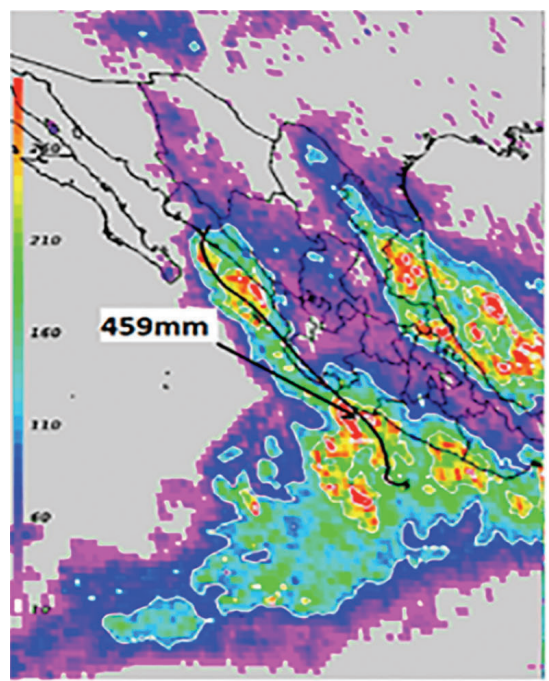

a)

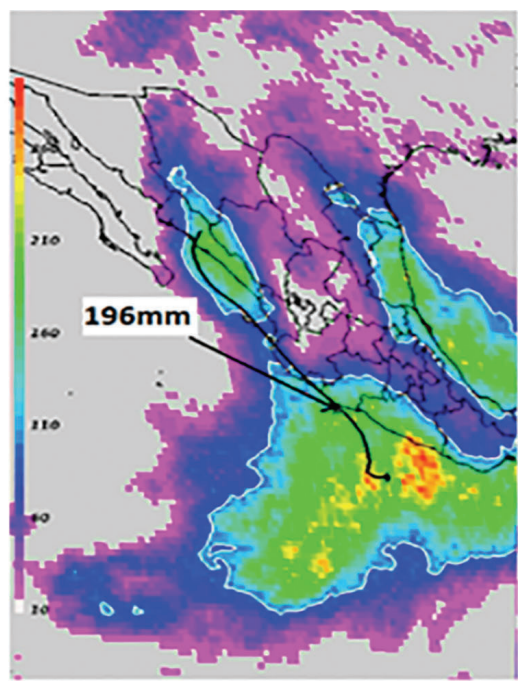

b)

Fuente: Elaboración propia usando IDv.

Figura 5. Estimación de lluvia usando a) TRMm y b) PERSiann para el huracán Manuel del 13 al 19 de septiembre de 2013 
Las estimaciones de TRMM y PERSIANN difieren en la forma en que se distribuyen los máximos de precipitación. TRMM aparentemente realiza mejores estimaciones que PERSIANN a pesar de que ambos productos tienen la misma resolución espacial. La forma en que se compararon ambas estimaciones fue utilizando los datos de lluvia registrados por los pluviómetros de la red de estaciones de superficie distribuidas por todo el país, y por efectos de trabajo de investigación se consideraron todas las estaciones en los estados del lado del Pacífico mexicano. Se encontró que ambos sistemas subestiman la lluvia registrada por la red de estaciones meteorológicas de superficie: TRMm la subestima aproximadamente $39 \%$ y PERSIANN alrededor de $46 \%$.

\section{Patricia (2015)}

Debido a que trmm ya fue destruido y no se cuenta con más datos del producto satelital se realizó una última estimación usando el producto TRMм en tiempo real, y desafortunadamente aún no hay datos disponibles en PERSIANN. La figura 7 muestra la estimación de lluvia hecha por el nuevo producto.

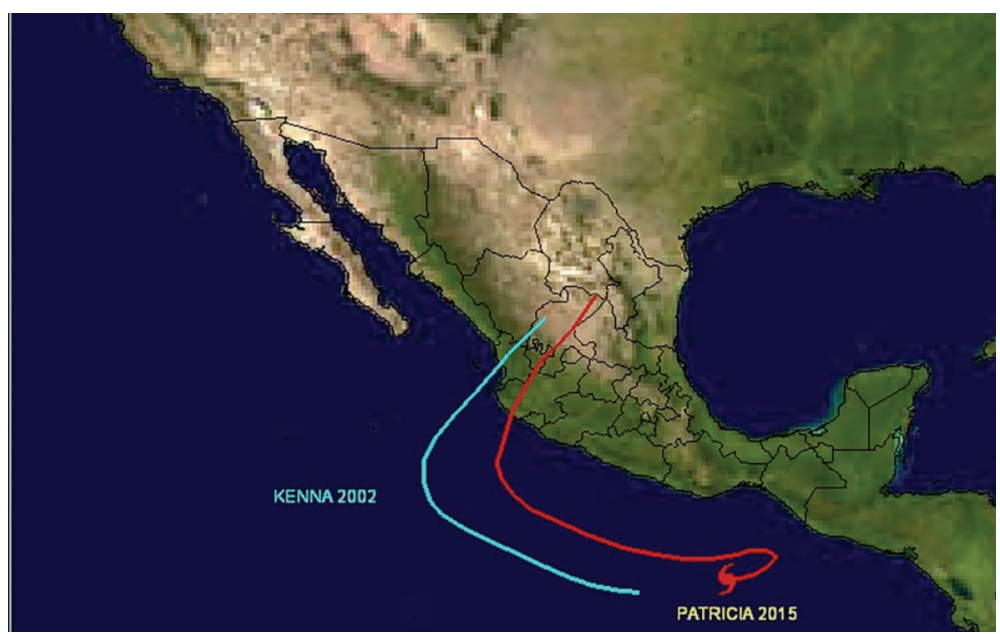

Fuente: Elaboración propia usando IDv.

FiguRa 6. Trayectorias observadas de Kenna (22 a 26 de octubre de 2002) y Patricia (22 a 24 de octubre de 2015) 


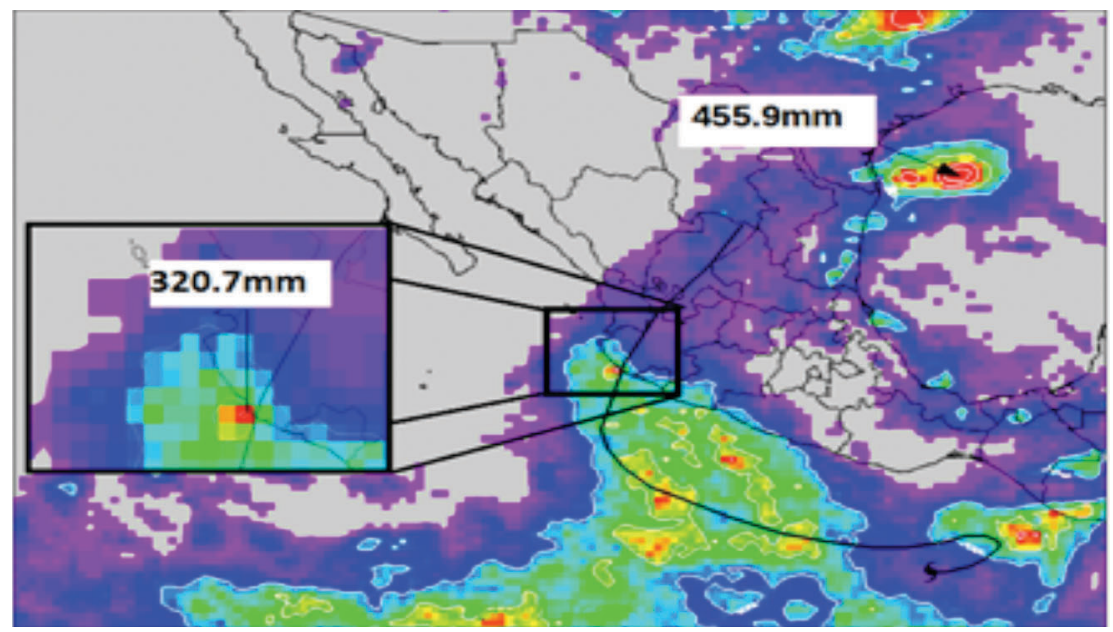

Fuente: Elaboración propia usando datos de тRмм.

Figura 7. Estimación de lluvia mediante el producto trmm 3B42RTv7. Las fechas consideradas son 22 al 24 de octubre del 2015

Si bien Patricia ocasionó severas pérdidas económicas a los pobladores en los lugares afectados, el daño no fue tan grande como se esperaba, ya que la orografía de Jalisco pudo haber ejercido fuerzas friccionales sobre el huracán que ocasionaron que se degradara a тт. La figura 7 muestra los máximos de lluvia registrados por las estaciones meteorológicas cercanas al paso del hidrometeoro.

En las figuras presentadas, las estimaciones de TRMM y PERSIANN tienen los mismos intervalos de contornos (mín $=100$, máx $=500$ ) y se puede apreciar que TRMM realiza mejor estimación que PERSIANN tan solo observando los casos considerados.

Cabe resaltar el problema de actualización de datos de las estaciones climatológicas del smn que están disponibles al público. La mayoría de las estaciones revisadas para la zona costera del Pacífico mexicano tiene datos disponibles hasta 2010, y hubo algunos casos donde las estaciones están clasificadas como “en operación” pero sin datos, probablemente por algún desperfecto. Sin embargo, los datos de сцicom han sido de gran utilidad para cubrir la información faltante, ya que es una base de datos muy extensa que ha sido adoptada por el 
SMN para publicar los datos registrados por la gran red de estaciones meteorológicas de superficie distribuidas por todo el territorio mexicano. En el Centro de Investigación Científica y de Educación Superior de Ensenada, Baja California -CICESE- (http://clicom-mex.cicese.mx) se pueden visualizar y descargar datos de las estaciones cuicom y de ст.

\section{Pronósticos oficiales}

Para la observación y evaluación del pronóstico de trayectoria e intensidad de los Ст se requirió de la base de datos de Errores de Trayectoria e Intensidad del Pronóstico Oficial del NHc, la cual está compuesta de los errores desde la hora 0 hasta las 120 horas uTc (tiempo universal coordinado, por sus siglas en inglés). Tanto los errores de trayectoria como de intensidad son considerados como la distancia entre la posición del pronóstico y la observación desde la hora 0 hasta las 120 horas, mientras que el error de intensidad es la diferencia entre la magnitud del viento máximo sostenido pronosticado y el viento dado por la mejor trayectoria.

También se requirió de la trayectoria observada del evento ciclónico y las trayectorias del OFCL y del pronóstico GFs. El ofCL es resultado de la experiencia humana, el pronosticador evalúa los distintos modelos, además del rendimiento actual e histórico del modelo, así como de su experiencia.

El GFs constituye un sistema global de predicción del clima, configurado por cuatro modelos: atmosférico, oceánico, tierra y mar-hielo, que en conjunto proveen una mejor representación de las condiciones atmosféricas. A través de este modelo se encuentra disponible una amplia gama de variables atmosféricas, como viento, altura geopotencial, precipitación, humedad, entre otras.

Se determinó la habilidad (skill) del pronóstico ofcL con respecto al pronóstico estadístico CLIPER como punto de referencia para evaluar la habilidad de cualquier pronóstico (DeMaria y Gross, 2003):

$$
\mathrm{ER}=100\left(\mathrm{E}_{\text {modelo }}-\mathrm{E}_{\text {CLIPER }}\right) / \mathrm{E}_{\text {CLIPER }}
$$

Donde ER es el error relativo expresado en porcentaje, $\mathrm{E}_{\text {modelo }}$ es el error del modelo a evaluar y $\mathrm{E}_{\text {CLIPER }}$ es el error dado por el pronóstico CLIPER. Los valores negativos representan una mejor habilidad del pronóstico que se está evaluando, mientras que los valores positivos, lo contrario. 
Finalmente, para conocer el comportamiento de las capas altas de la atmósfera se elaboraron mapas de altura geopotencial a 500 milibares (mb) tanto del pronóstico GFs como de lo observado, considerando la fecha/hora en que la trayectoria del ciclón mostró mayor error.

A continuación se exponen los errores del pronóstico ofCL para los ciclones estudiados.

\section{Jova (2011)}

El cuadro 1 presenta los errores promedio de trayectoria e intensidad desde la hora 0 hasta la hora 120 de pronóstico de Jova. Los errores de trayectoria se incrementaron con el paso del tiempo. Sin embargo, a la hora 96 del pronóstico se observó un máximo de $379 \mathrm{~km}$ y posteriormente un decremento del error. Este error rebasó el promedio de la hora 96. También se advirtió que en el máximo error de trayectoria de la hora 96 el error de intensidad fue 0.

CUADRo 1. Errores promedios de Trayectoria e Intensidad de Jova, desde la hora 0 a la 120 horas de pronóstico, en paréntesis número de pronóstico utilizados

\begin{tabular}{lcccccccc}
\multicolumn{1}{c}{ Hora } & 0 & 12 & 24 & 36 & 48 & 72 & 96 & 120 \\
$\begin{array}{l}\text { Trayectoria } \\
(\mathrm{km})\end{array}$ & $21(28)$ & $55(26)$ & $72(24)$ & $81(22)$ & $100(20)$ & $148(16)$ & $244(12)$ & $238(5)$ \\
$\begin{array}{l}\text { Intensidad } \\
(\mathrm{m} / \mathrm{s})\end{array}$ & $0.09(28)$ & $0.59(26)$ & $0.3(24)$ & $0.35(22)$ & $1.7(20)$ & $-0.5(16)$ & $-17(12)$ & $-23(5)$ \\
$\begin{array}{l}\text { Máximo error } \\
\text { de Trayectoria }\end{array}$ & 111 & 157 & 226 & 317 & 377 & 268 & 379 & 350 \\
$\begin{array}{l}\text { Máximo error } \\
\text { de Intensidad }\end{array}$ & 8 & 13 & 15 & 13 & 15 & 10 & 0 & -15
\end{tabular}

Fuente. Elaboración propia con datos del $\mathrm{CNH}$

Al calcular el error relativo que expresa la habilidad de un pronóstico, que para este caso es el pronóstico ofcL respecto al pronóstico base CLIPER, se encontró que el ofcL presentó baja habilidad (figura 8) durante las primeras horas de pronóstico respecto al CLIPER. 


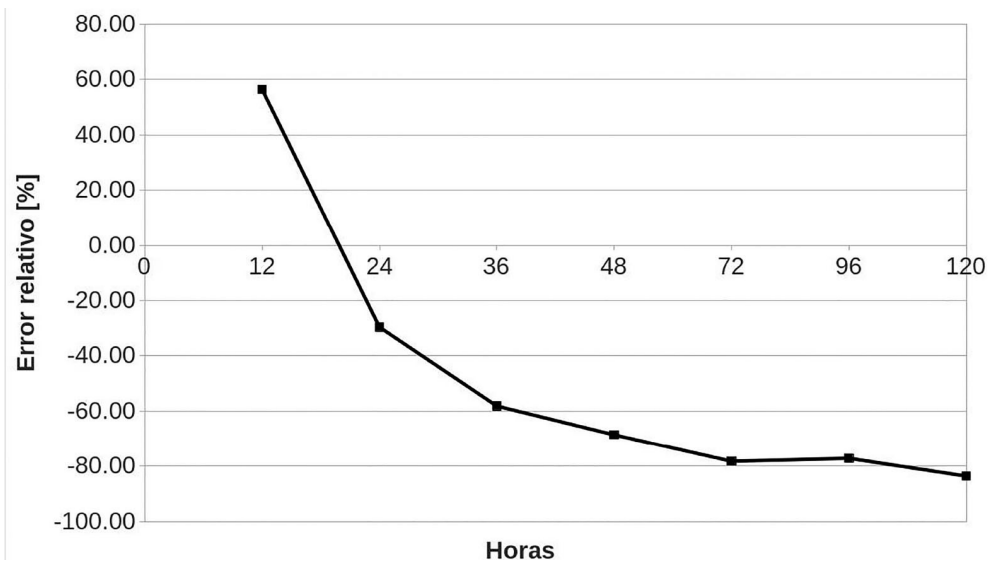

Fuente. Elaboración propia con datos del CNH.

FiguRA 8. Error relativo del pronóstico ofcl versus pronóstico cliper para el ciclón tropical Jova que se desarrolló del 6 al 12 de octubre de 2011

Por otro lado, en la figura 9 se muestra el comportamiento de la atmósfera durante el máximo error a la hora 96 de pronóstico. Se observó un comportamiento zonal hacia el oeste. Aunque el pronóstico subestimó el comportamiento de la atmósfera, definió los centros ciclónicos cerca del desarrollo de Jova.

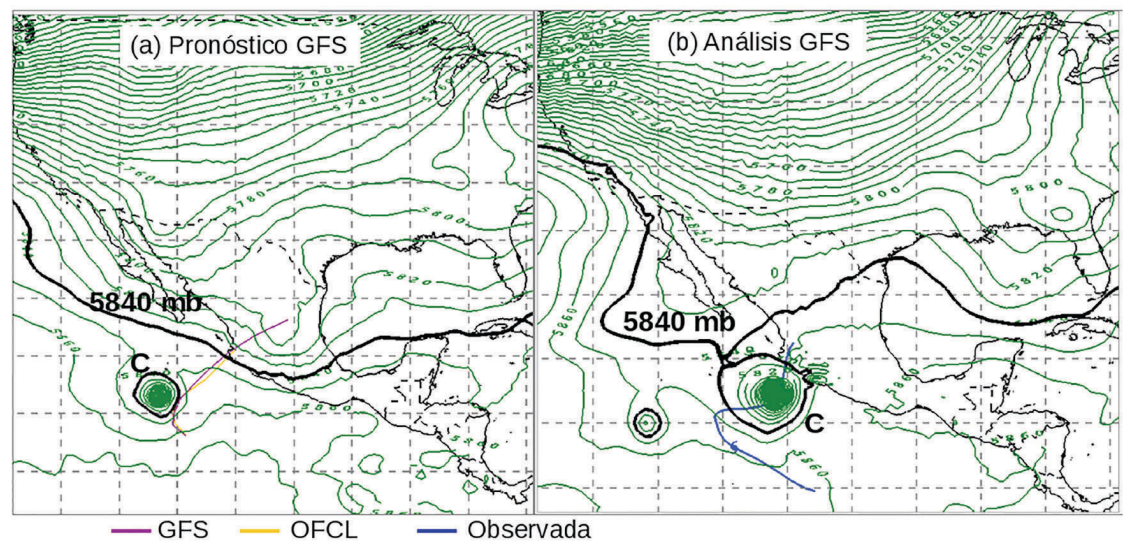

Fuente: Elaboración propia usando IDv.

FiguRA 9a, b. Altura geopotencial a 500 mb del pronóstico GFs (7 de octubre a las 6 horas) y el análisis GFs ( 11 de octubre a las 6 horas) del ст Jova 


\section{Manuel (2013)}

Manuel tuvo un menor número de pronósticos (de la 0 a la hora 48 de pronóstico) en comparación con Jova. El cuadro 2 expone los errores de trayectoria e intensidad de Manuel. Para el 15 de octubre, horas antes de tocar tierra, el error de trayectoria a las $24 \mathrm{~h}$ era de $87 \mathrm{~km}$ con respecto a la trayectoria observada.

CUADRo 2. Errores promedios de Trayectoria e Intensidad de Manuel, desde la hora 0 a la 120 horas de pronóstico, en paréntesis número de pronóstico utilizados

\begin{tabular}{lcccccccc}
\multicolumn{1}{c}{ Hora } & 0 & 12 & 24 & 36 & 48 & 72 & 96 & 120 \\
$\begin{array}{l}\text { Trayectoria } \\
(\mathrm{km})\end{array}$ & $18(20)$ & $43(16)$ & $70(12)$ & $99(8)$ & $145(4)$ & $\begin{array}{c}\text { No } \\
\text { hubo }\end{array}$ & $\begin{array}{c}\text { No } \\
\text { hubo }\end{array}$ & $\begin{array}{c}\text { No } \\
\text { hubo }\end{array}$ \\
$\begin{array}{l}\text { Intensidad } \\
\text { (m/s) }\end{array}$ & $-0.4(20)$ & $-1.1(16)$ & $-3.4(12)$ & $-3.2(8)$ & $-4.5(4)$ & $\begin{array}{c}\text { No } \\
\text { hubo }\end{array}$ & $\begin{array}{c}\text { No } \\
\text { hubo }\end{array}$ & $\begin{array}{c}\text { No } \\
\text { hubo }\end{array}$ \\
$\begin{array}{l}\text { Máximo error } \\
\text { de Trayectoria }\end{array}$ & 46 & 109 & 119 & 204 & 258 & --- & --- & --- \\
$\begin{array}{l}\text { Máximo error } \\
\text { de Intensidad }\end{array}$ & 7.5 & 10 & 18 & 5 & 5 & --- & --- & --- \\
\hline
\end{tabular}

Fuente: Elaboración propia con datos del cNH.

En la figura 10 se muestra la habilidad del pronóstico ofCL; en este caso, la habilidad es débil para las primeras horas de pronóstico, después aumentó ligeramente. En comparación con Jova, el pronóstico ofcL para Manuel tuvo menor habilidad.

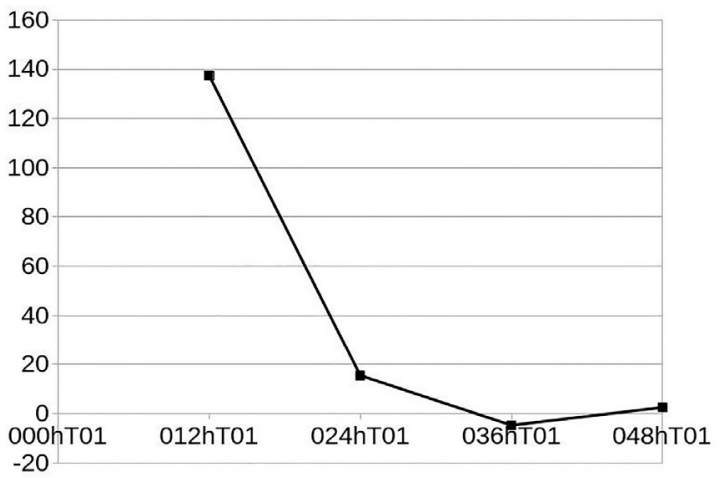

Fuente: Elaboración propia con datos del cNH. para el ciclón tropical Manuel del 13 al 19 de septiembre de 2013 


\section{Patricia (2015)}

Para este ciclón aún no se tiene información de los errores de trayectoria e intensidad. Sin embargo, en la figura 11 se indica la trayectoria observada y las trayectorias de los pronósticos GFS y OFCL para el día en que tocó tierra sobre las costas de Jalisco (23 de octubre a las 18:15 hora local). Se observó que para esa hora los pronósticos mostraron buena aproximación.

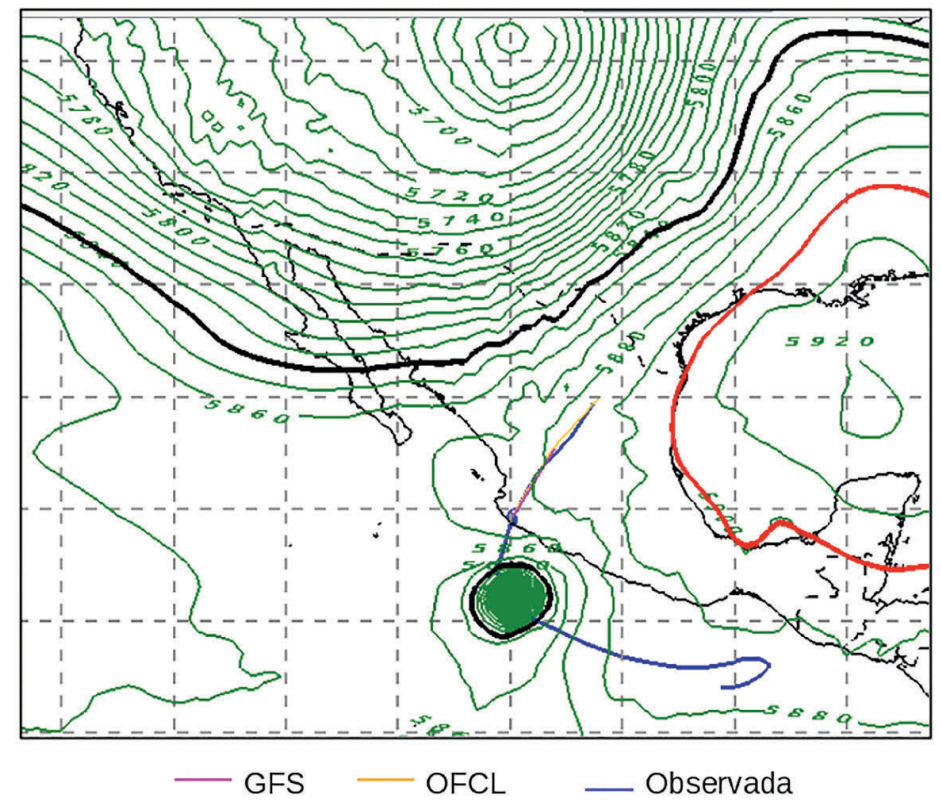

Fuente: Elaboración propia en el software IDV.

Figura 11. Trayectoria del ciclón tropical Patricia del pronóstico gfs, OFCL y observación (23 de octubre a las 18:15 hora local)

En la figura 12 se aprecia el comportamiento de los pronósticos de intensidad de Patricia: OFCL, GFS, SHIFOR (modelo estadístico de intensidad) y HWRF. Aunque no se cuenta con los errores de intensidad, se advirtió que ninguno de los pronósticos tuvo una buena aproximación. En el momento en que Patricia tocó tierra, el 23 de octubre a las 18:15 hora local, presentó una velocidad máxima sostenida observada de $259.2 \mathrm{~km} / \mathrm{h}$, mientras que lo emitido (24 horas antes) por el pronóstico ofcl fue de $185 \mathrm{~km} / \mathrm{h}$. 


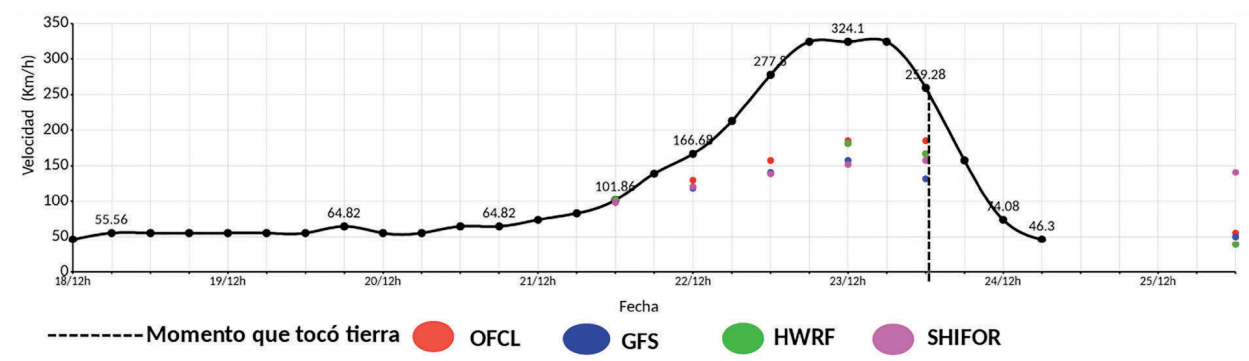

Fuente: Elaboración propia.

FiguRa 12. Pronósticos de intensidad para el ciclón tropical Patricia

\section{Discusiones}

Los resultados de comparar las estimaciones de los dos sistemas de percepción remota evidenciaron que tanto TRMM como PERSIANN subestiman la cantidad de precipitación asociada a los cт.

La combinación de varias imágenes fue útil para observar cómo se distribuyó la nubosidad asociada a los ст durante su evolución espaciotemporal, así como los lugares donde se produjo la convección profunda y precipitaciones puntuales muy intensas, especialmente sobre tierra firme.

Antes de las 24 horas del pronóstico, el ofcL no tiene buena habilidad con relación al CLIPER; esto se debe a que este último es un modelo estadístico de persistencia, el cual predice los movimientos del ciclón a intervalos de 12 horas hasta 120 horas y considera para su predicción la posición actual y el movimiento 12 horas antes, lo que hace que en las primeras horas el pronóstico CLIPER tenga buenos resultados. De las ocho horas de pronóstico (0, 12, 24, 36, 48, 72, 96 y 120) la mayor habilidad se presentó a las 48 y 72 horas.

\section{Conclusiones}

Se hizo la comparación entre las estimaciones de lluvia asociada a ст para la franja costera, incluyendo al estado de Jalisco, y se encontró que tanto TRMM como PERSIANn subestiman la cantidad de lluvia que registraron las estaciones meteorológicas de superficie, teniendo TRMM un mejor desempeño que PERSIANN. 
Se combinaron las imágenes infrarrojas de los satélites goEs para observar la distribución y propiedades de la nubosidad asociada a los ст considerados; de esta forma se pudo determinar que fue en el mar donde se produjo la mayor convección asociada a los casos seleccionados. También se puede decir que la nubosidad en las imágenes infrarrojas coincidió de manera razonable con la de las imágenes de las estimaciones de TRMM y un poco menos con PERSIANN.

Se logró identificar los lugares donde se produjo la precipitación más intensa por medio de las temperaturas de los topes de las nubes más altas, pues al tener menor temperatura esos topes, significa que las tormentas se desarrollan a mayor altura en la tropósfera y también son los sitios donde se generan las lluvias más intensas.

La información recabada permitió evaluar el pronóstico ofcL tanto de trayectoria como de intensidad. Se encontró que los errores de trayectoria aumentan con el tiempo, sin embargo, los errores de intensidad no se comportan así. Además, la intensidad es más difícil de pronosticar y en ocasiones pueden existir grandes diferencias entre lo observado y lo pronosticado, como en el caso de Patricia, en el que la intensidad pronosticada estuvo por debajo de lo observado. Sin embargo, la trayectoria dada por el pronóstico ofcL para este ciclón a la hora en que tocó tierra sobre Jalisco presentó un comportamiento similar a la trayectoria observada.

\section{Fuentes consultadas}

Anthes, R. A. (1982). Tropical Cyclones: Their Evolution, Structure and Effects. Boston: American Meteorological Society.

Bravo Lujano, C. y Hernández Unzón, A. (2011). Reseña del Huracán “Jova” del Océano Pacífico. Recuperado de http://smn.cna.gob.mx/ciclones/ tempo2011/pacifico/Jova-p11.pdf

Brennan, M. J. (2012). Tropical Cyclone Report, Hurricane Jova. Retrieved from http://www.nhc.noaa.gov/data/tcr/EP102011_Jova.pdf [2016, January $9^{\text {th }}$.

Colín Olivares, O., Chimal Vázquez, A. E. e Ihl, T. (2015). Fenómenos hidrometeorológicos extremos en ciudades turísticas del norte de Quintana Roo. Teoría y Praxis, núm. esp., 84-98. 
DeMaria, M. y Gross, J. M. (2003). Evolution of Prediction Models. En R. Simpson, R. Anthes, M. Garstang y J. Simpson (eds.), Hurricane! Coping with Disaster: Progress and Challenges Since Galveston, 1900 (pp. 103126). Washington, D. C.: American Geophysical Union.

Ebert, E. y Holland, G. (1990). Observations of Cold Cloud-Top Temperatures in Tropical Cyclone Hilda (1990). Monthly Weather Review, 120, 22402251.

Farfán, L. M., D’Sa, E. J., Liu, K. y Rivera-Monroy, V. (2014). Tropical Cyclone Impacts on Coastal Regions: the Case of the Yucatán and the Baja California Peninsulas, México. Estuaries and Coasts, 37, 1388-1402.

Heming, J. y Goerss, J. (2010). Track and structure forecasts of tropical cyclones. En J. C. L. Chan y J. D. Kepert (eds.), Global Perspectives on Tropical Cyclones: From Science to Mitigation (pp. 287-323). Singapur: World Scientific.

Kimberlain, T. B., Blake, E. S. y Cangialosi, J. P. (2016). Hurricane Patricia. Retrieved from http://www.nhc.noaa.gov/data/tcr/EP202015_Patricia.pdf

Nájar, A. (25 de octubre de 2015). Los pueblos de México donde tocó tierra Patricia, "el huracán más poderoso de la historia". BBC Mundo. Recuperado de http://www.bbc.com/mundo/noticias/2015/10/151025_ huracan_patricia_pueblos_mexico_an [2015, 3 de enero].

Partida Bush, V. (2006). Proyecciones de la población de México 2005-2050. México: Consejo Nacional de Población. Recuperado de http://www. conapo.gob.mx/work/models/CONAPO/proyecciones_estatales/Proy05-50.pdf

Pasch, R. J. y Zelinsky, D. A. (2014). Hurricane Manuel. Retrieved from http:// www.nhc.noaa.gov/data/tcr/EP132013_Manuel.pdf [2016, January $\left.9^{\text {th }}\right]$.

Rosengaus, M. (2001). Análisis de la temporada 2000 de ciclones tropicales en México. Ingeniería Hidráulica en México, XVI(4), 5-23.

Roy, Ch. y Kovordányi, R. (2012). Tropical cyclone track forecasting techniquesA review. Atmospheric Research, 104-105, 40-69. 